\title{
Effect of vacuum polarization of charged massive fermions in an Aharonov-Bohm field
}

\author{
V. R. Khalilov ${ }^{\mathrm{a}}$ \\ Faculty of Physics, M.V. Lomonosov Moscow State University, 119991 Moscow, Russia
}

Received: 3 July 2014 / Accepted: 28 August 2014 / Published online: 16 September 2014

(c) The Author(s) 2014. This article is published with open access at Springerlink.com

\begin{abstract}
The effect of vacuum polarization of charged massive fermions in an Aharonov-Bohm (AB) potential in 2 +1 dimensions is investigated. The causal Green function of the Dirac equation with the $\mathrm{AB}$ potential is represented via the regular and irregular solutions of the two-dimensional radial Dirac equation. It is shown that the vacuum current density contains the contribution from free filled states of the negative energy continuum as well as that from a bound unfilled state, which can emerge in the above background due to the interaction of the fermion's spin magnetic moment with the $\mathrm{AB}$ magnetic field, while the induced charge density contains only the contribution from the bound state. The expressions for the vacuum charge and induced current densities are obtained (recovered for massless fermions) for the graphene in the field of infinitesimally thin solenoid perpendicular to the plane of a sample. We also find the bound state energy as a function of magnetic flux, fermion spin, and the radius of solenoid, and we discuss the role of the so-called self-adjoint extension parameter and determine it in terms of the physics of the problem.
\end{abstract}

\section{Introduction}

Quantum systems of relativistic fermions in external fields in $2+1$ dimensions attract considerable interest, which is related to the possibility of applying the results obtained for simplified models to the study of fundamental physical phenomena such as the Aharonov-Bohm and quantum Hall effects [1,2], as well as high-temperature superconductivity [3]. When the external field configuration has the cylindrical symmetry, a natural assumption is that the relevant quantummechanical system is invariant along the symmetry $(z)$ axis and the system then becomes essentially two-dimensional in the $x y$ plane. Solutions of the Dirac equation with the AB

a e-mail: khalilov@phys.msu.ru potential in $2+1$ dimensions have been used to describe scattering of spin-polarized electrons by infinitesimally thin solenoid [4] as well as the behavior of fermions in the field of a cosmic string [5].

Important physical quantities are the vacuum charge and current densities induced by the background field. Vacuum polarization effects in the $(2+1)$-dimensional quantum electrodynamics with a homogeneous magnetic field and with a nonzero fermion density were studied in $[6,7]$.

Interest to two-dimensional fermion systems with the energy spectrum governed by Dirac Hamiltonian was revived in connection with the problems of graphene (see, e.g., [811]). In graphene, the electrons near the Fermi surface can be described in terms of an effective Lorentz-invariant theory, with their kinetic energy determined by Dirac's dispersion law, and the dynamics of the electron at low energies is described by the Dirac equation in $2+1$ dimensions for a zero-mass fermion [9]. It should be noted that, while a description of electron states in graphene in [12-14] was based on the Dirac equation for massless fermions, Ref. [15] has shown that the massive case can also be created.

At the same time, the effective fine structure constant in graphene is large, which leads to a new possibility for studying quantum electrodynamics in the strong-coupling regime. Charged impurity screening in graphene in terms of vacuum polarization was investigated in [14,16-19]. The induced vacuum current in graphene in the field of a solenoid was investigated in [20] and a wonderful phenomenon was revealed: the induced current turns out to be a finite periodic function of the magnetic flux. Induced vacuum condensates such as the induced charge density and current (and other ones) for massless fermions in the background of a singular magnetic vortex in $(2+1)$-dimensional space-time were investigated in [21,22].

The Dirac equation with the AB potential is governed by a (singular) Hamiltonian that requires the supplementary definition in order for it to be treated as a self-adjoint quantum- 
mechanical operator. Based on the form asymmetry selfadjoint extension method [23,24], the most relevant physical quantities, such as energy spectrum, wave functions, and the self-adjoint extension parameter, were determined for some quantum systems with the $\mathrm{AB}$ potential by applying the socalled self-adjoint boundary conditions in [25-28]. It is useful to note that the self-adjoint extension regularization was applied for two-dimensional models in quantum field theory (see $[29,30])$. As a result some uncertainty appears in the prediction of physical quantities [29,30]. Essentially, a physical interpretation of self-adjoint extensions is a purely physical problem and each extension can (and must) be understood through an appropriate physical regularization [24].

In the present paper, we use the physical regularization method for constructing the fermion wave function and determining the energy in a bound state as a function of magnetic flux and fermion spin. We introduce a small parameter $R$, which, if we imagine a real solenoid, is the finite radius of this solenoid (see, for instance, [4,31,32].

We shall adopt the units where $c=\hbar=1$.

\section{Solutions to the Dirac equation in an Aharonov-Bohm field}

The space of particle quantum states in two spatial dimensions is the Hilbert space $\mathfrak{H}=L^{2}\left(\mathbb{R}^{2}\right)$ of square-integrable functions $\Psi(\mathbf{r}), \mathbf{r}=(x, y)$ with the scalar product

$\left(\Psi_{1}, \Psi_{2}\right)=\int \Psi_{1}^{\dagger}(\mathbf{r}) \Psi_{2}(\mathbf{r}) \mathrm{d} \mathbf{r}, \quad \mathrm{d} \mathbf{r}=\mathrm{d} x \mathrm{~d} y$.

The Dirac equation for a fermion in a given external field can be obtained just as in $3+1$ dimensions. The Dirac $\gamma^{\mu}$ matrix algebra is known to be representable in terms of the two-dimensional Pauli matrices $\sigma_{j}$ :

$\gamma^{0}=\sigma_{3}, \quad \gamma^{1}=i s \sigma_{1}, \quad \gamma^{2}=i \sigma_{2}$

and the parameter $s= \pm 1$ can be introduced to label two types of fermions in accordance with the signature of the twodimensional Dirac matrices [33] and is applied to the characterization of the two states of the fermion spin (spin "up" and "down") [4]. Then the Dirac Hamiltonian for a fermion of the mass $m$ and charge $e=-e_{0}<0$ in an AharonovBohm potential $A_{0}=0, \quad A_{r}=0, \quad A_{\varphi}=B / r, \quad r=$ $\sqrt{x^{2}+y^{2}}, \quad \varphi=\arctan (y / x)$ is

$H_{D}=\sigma_{1} P_{2}-s \sigma_{2} P_{1}+\sigma_{3} m$,

where $P_{\mu}=-i \partial_{\mu}-e A_{\mu}$ is the generalized fermion momentum operator (a three-vector). The Hamiltonian (3) should be defined as a self-adjoint operator in the Hilbert space of square-integrable two-spinors $\Psi(\mathbf{r})$ with the scalar product (1). The total angular momentum $J \equiv L_{z}+s \sigma_{3} / 2$, where $L_{z} \equiv-i \partial / \partial \varphi$, commutes with $H_{D}$.
In the real physical space, because of the existence of finite magnetic flux inside the solenoid $\Phi=2 \pi B$ the term including the spin parameter appears in the form of an additional delta-function interaction of the spin with the magnetic field of the solenoid,

$\mathbf{H}=(0,0, H)=\nabla \times \mathbf{A}=B \pi \delta(\mathbf{r})$

in the Dirac equation squared. The additional potential

$-\operatorname{seB} \frac{\delta(r)}{r}$

will be taken into account by boundary conditions. Such a kind of point interaction also appears in several AharonovBohm-like problems [34-37].

The eigenfunctions of the Hamiltonian (3) are (see [38])

$\Psi(t, \mathbf{r})=\frac{1}{\sqrt{2 \pi}}\left(\begin{array}{l}f_{1}(r) \\ f_{2}(r) e^{i s \varphi}\end{array}\right) \exp (-i E t+i l \varphi)$,

where $E$ is the fermion energy and $l$ is an integer. The wave function $\Psi(t, \mathbf{r})$ is an eigenfunction of the operator $J$ with eigenvalue $j=l+s / 2$. Taking into account the easily checked relations

$s P_{1} \pm i P_{2}=-i e^{ \pm i s \varphi}\left[s \frac{\partial}{\partial r} \pm\left(\frac{i}{r} \frac{\partial}{\partial \varphi}-\frac{\mu}{r}\right)\right]$,

where $\mu \equiv e_{0} B$, we reduce the problem to that for the radial Hamiltonian $\breve{h}$ in the Hilbert space of doublets $F(r)$ squareintegrable on the half-line:

$\breve{h} F=E F, F=\left(\begin{array}{l}f_{1}(r) \\ f_{2}(r)\end{array}\right)$,

where

$$
\begin{aligned}
\check{h}\left(\begin{array}{l}
f_{1}(r) \\
f_{2}(r)
\end{array}\right) \\
=\left[\begin{array}{ll}
m & s d / d r+(l+\mu) / r \\
-s d / d r+(l+\mu+s) / r & -m
\end{array}\right] \\
\quad \times\left(\begin{array}{l}
f_{1}(r) \\
f_{2}(r)
\end{array}\right)=E\left(\begin{array}{l}
f_{1}(r) \\
f_{2}(r)
\end{array}\right)
\end{aligned}
$$

in the range $r>0$. Eliminating, for instance, $f_{2}(r)$ we derive the differential equation for $f_{1}(r)$ and then the lower ("small") component of doublet is found from the relation

$f_{2}(r)=-\frac{1}{E+m}\left(s \frac{\mathrm{d} f_{1}}{\mathrm{~d} r}-\frac{l+\mu}{r} f_{1}\right)$.

Then for $E^{2}-m^{2}>0$ the radial solutions can be written via the Bessel functions:

$F(r, E)=\left(\begin{array}{l}f_{1} \\ f_{2}\end{array}\right)=A\left(\begin{array}{l}\sqrt{E+m} J_{v}(p r) \\ \pm \sqrt{E-m} J_{v \pm s}(p r)\end{array}\right)$.

Here $A$ is a constant, $v=|l+\mu|, p=\sqrt{E^{2}-m^{2}}$ and the upper (lower) signs should be taken for $l+\mu>0(l+\mu<0)$. 
Let us represent

$\mu=[\mu]+\beta \equiv n+\beta$,

where $n$ denotes the largest integer $\leq \mu$, i.e. the integer part of $\mu$, and $0<\beta<1$ is the fractional part of $\mu$. Hence $n=0,1,2, \ldots$ for $\mu>0$ and $n=-1,-2,-3, \ldots$ for $\mu<0$. Note that the signs of $e$ and $B$ can be fixed and the potential is invariant under the changes $e \rightarrow-e, s \rightarrow-s$; it hence suffices to consider only the case $e<0, \mu>0$. One can suppose that a bound state exists due to the interaction of the fermion spin magnetic moment with $\mathrm{AB}$ magnetic field, which must be attractive. In the case $\mu>0$, the potential is attractive for $s=-1$ and repulsive for $s=1$, in the case $\mu<0$; it is attractive for $s=1$ and repulsive for $s=-1$. Then it is seen that when $\beta \neq 0$ the upper and lower components of (11) are integrable near $r=0$ only for $v \pm s>-1$.

A linearly independent solution at $v \neq 0, U(r ; E)$, is determined as follows:

$U(r, E)=B\left(\begin{array}{l}\sqrt{E+m} J_{-v}(p r) \\ \pm \sqrt{E-m} J_{-\nu \mp s}(p r)\end{array}\right)$.

Here $B$ is a constant. We shall need the irregular (i.e. integrable at $r \rightarrow \infty$ ) solutions for $E^{2}-m^{2}<0$. Such irregular solutions, nontrivial at $v \neq n / 2, n=1,2, \ldots$ are a linear combination of $F$ and $U$ and also can be represented via the MacDonald functions:

$V(r, E)=\left(\begin{array}{l}v_{1} \\ v_{2}\end{array}\right)=B\left(\begin{array}{l}\sqrt{m+E} K_{v}(\lambda r) \\ \pm s \sqrt{m-E} K_{v \pm s}(\lambda r)\end{array}\right)$,

where $C$ is a constant and $\lambda=\sqrt{m^{2}-E^{2}}$. The irregular solutions are integrable at $r \rightarrow \infty$.

\section{Bound fermion state energy: physical regularization}

A $\delta(x)$ potential is convenient to take into account artificially by means of the continuity relations. For this we replace the spin term (5) by [4]

$-\operatorname{se} B \frac{\delta(r-R)}{R}$

and take account of it by means of the continuity relations in $R$. The quantity $B \delta(r-R) / R$ should not be considered as the real magnetic field inside a flux tube but only as a field model allowing us to take it it into account by means of the continuity relations for solutions in two ranges. Although the functional structures of Eqs. (5) and (15) are quite different, as discussed in [32], we are free to use any form of potential, provided that only the contribution of the form (5) is excluded.

Now we can find the wave function and the energy of the bound state by means of solutions to the Dirac equation in the ranges $r<R$ and $r>R$ and the potential (15) can be taken into account by means of the continuity relations. Obviously, for the model with the zero AB potential in the range $r<R$ the radial solutions must satisfy (9) at $\mu=0$. They are written via the Bessel functions, integrable near $r=0$ :

$S(r, E)=\left(\begin{array}{l}s_{1} \\ s_{2}\end{array}\right)=C\left(\begin{array}{l}\sqrt{E+m} J_{|l|}(p r) \\ \pm \sqrt{E-m} J_{|l| \pm s}(p r)\end{array}\right)$,

where $C$ is a constant, $|l| \pm s \geq 0$ and the upper (lower) signs should be taken for $l>0(l<0)$. The continuity relations can be written as

$$
\left(\begin{array}{l}
s_{1} \\
s_{2}
\end{array}\right)_{R-\delta}=\left(\begin{array}{l}
v_{1} \\
v_{2}
\end{array}\right)_{R-\delta}, \quad \delta \rightarrow 0 .
$$

The left- and right-hand sides of (17) are calculated using the asymptotic representation for the Bessel functions in the limit $z \ll 1$ :

$$
\begin{aligned}
& J_{v}(z)=\frac{z^{v}}{2^{v} \Gamma(1+v)}, \\
& K_{v}(z)=-\frac{\pi}{2 \sin (\pi v)}\left[\frac{z^{v}}{2^{v} \Gamma(1+v)}-\frac{z^{-v}}{2^{-v} \Gamma(1-v)}\right] .
\end{aligned}
$$

Here $\Gamma(x)$ is the Euler gamma function of argument $x$.

The energy spectrum is determined by

$$
\left(\frac{s_{1}}{s_{2}}\right)_{R-\delta}=\left(\frac{v_{1}}{v_{2}}\right)_{R-\delta}, \quad \delta \rightarrow 0 .
$$

As a result, we obtain

$$
\begin{aligned}
-s & \sqrt{\frac{E-m}{m-E}} \frac{(k R)^{ \pm s} \Gamma(1+|l|)}{2^{ \pm s} \Gamma(1+|l| \pm s)} \\
= & {\left[\frac{(\lambda R)^{v \pm s}}{2^{v \pm s} \Gamma(1+v \pm s)}-\frac{(\lambda R)^{-(v \pm s)}}{2^{-(v \pm s)} \Gamma(1-(v \pm s))}\right] } \\
& \times\left[\frac{(\lambda R)^{v}}{2^{v} \Gamma(1+v)}-\frac{(\lambda R)^{-v}}{2^{-v} \Gamma(1-v)}\right]^{-1} .
\end{aligned}
$$

It follows from Eq. (20) that the bound state energy is determined by the poles of the gamma function $\Gamma(1+|l| \pm s)$ at $l=0$ :

$$
\begin{aligned}
& \frac{(\lambda R)^{-(n+\beta \pm s)}}{2^{-(n+\beta \pm s)} \Gamma(1-(n+\beta \pm s))}-\frac{(\lambda R)^{n+\beta \pm s}}{2^{n+\beta \pm s} \Gamma(1+n+\beta \pm s)} \\
& \quad=1 \pm s,
\end{aligned}
$$

where we must put $n=0$ and take the upper (lower) signs for $s=-1, \beta>0(s=1, \beta<0)$. As a result, we obtain the same equation for these two cases in the form

$\lambda=\frac{2}{R}\left(\frac{\Gamma(|\beta|)}{\Gamma(2-|\beta|)}\right)^{2(|\beta|-1)}$.

We define the particle (antiparticle) bound states as states that tend to the upper (lower) continuous boundary $m(-m)$ upon adiabatically slow switching of the external field. Only 
one particle (antiparticle) bound state with $s=-1(s=1)$ emerges. It is seen that these equations hold for the case $l+n=\mp 1(s= \pm 1)$. One can find from (22) that an adiabatic increase of the magnetic flux $(\beta)$ from 0 to 1 lifts a particle (antiparticle) energy level $E=m \rightarrow E=-m$ ( $E=-m \rightarrow E=m$ ) and for $|\beta|=1 / 2$ the particle and antiparticle energies are equal $E= \pm \sqrt{m^{2}-R^{-2}}$, where the upper (lower) signs should be taken for particle (antiparticle). We believe that the doublet

$V_{0}(r, E)=N\left(\begin{array}{l}\sqrt{m+E} K_{\beta}(\lambda r) \\ s \sqrt{m-E} K_{1-\beta}(\lambda r)\end{array}\right)$

( $N$ is a normalization factor) correctly represents the particle radial wave function (with $s=-1$ ) in the bound state. It is evident that the wave function (23) is singular at $r=0$ but square integrable on the half-line $[0, \infty)$ with the measure $r \mathrm{~d} r$.

The bound state energy for the considered system was derived in [28] by means of the self-adjoint extension method in the form

$\lambda=2 m\left(-\frac{\Gamma(|\beta-1 / 2|+1 / 2)}{\xi \Gamma(1 / 2-|\beta-1 / 2|)}\right)^{-2(|\beta-1 / 2|)}$.

Here $\xi=\tan (\theta / 2)(2 \pi \geq \theta \geq 0)$ parameterizes the selfadjoint extensions of the radial Dirac Hamiltonian, which are different for various $\theta$ except for the two equivalent cases $\theta=0,2 \pi$ (or $\xi= \pm \infty$ ). We can determine the self-adjoint extension parameter in terms of the physics of the problem, i.e. the parameter $R$. By comparing Eqs. (22) and (22) we arrive, for example for $\beta>1 / 2$, at

$\xi=-(m R)^{2 \beta-1} \frac{\Gamma(2-\beta)}{\Gamma(\beta)}$.

\section{Vacuum charge and current densities}

Now we consider the densities of the vacuum electric charge and vacuum electric current due to the vacuum polarization. These quantities are determined by the three-vector $j_{\mu}(\mathbf{r})$, which is expressed via the Green function of the Dirac equation as follows:

$j_{\mu}(\mathbf{r})=-\frac{e}{2} \int_{C} \frac{\mathrm{d} E}{2 \pi i} \operatorname{tr} G\left(\mathbf{r}, \mathbf{r}^{\prime} ; E\right) \gamma_{\mu}$,

where $C$ is the path in the complex plane of $E$ enclosing all the singularities along the real axis $E$ depending upon the choice of the Fermi surface (we choose $E_{F}=-m$ ). The singularities of $G\left(\mathbf{r}, \mathbf{r}^{\prime} ; E\right)$ can be simple poles associated with the discrete spectrum (in the range $-m<E<m$ ), and two cuts $(-\infty,-m]$ and $[m, \infty)$ associated with the continuum spectrum in the ranges $|E| \geq m$. As was shown in [39]), for the partial Green function in a Coulomb field in $3+1$ dimensions, the path $C$ may be deformed to run along the singularities on the real $E$ axis as follows: $C=$ $C_{-}+C_{p}+C_{+}$, where $C_{-}$is the path along the negative real $E$ axis $(\operatorname{Re} E<0)$ from $-\infty$ to 0 turning around at $E=0$ with positive orientation; $C_{p}$ is a circle around the bound states' singularities with $-m<E<0$, and $C_{+}$is the path along the positive real $E$ axis $(\operatorname{Re} E>0)$ from $\infty$ to 0 but with negative orientation (i.e. clockwise path) turning around at $E=0$. We note that, due to the Furry theorem, the spatial component of the induced vacuum current in an $\mathrm{AB}$ potential in $2+1$ dimensions should be an odd function of $\mu$. In the case considered this is the $j_{\varphi}$-component of the induced vacuum current. It will be recalled that the $j_{\varphi}$-component is the vector product of the vectors $\mathbf{j}$ and $\mathbf{n}=\mathbf{r} / r$ and has the only component $[\mathbf{j} \times \mathbf{n}]=j_{x} n_{y}-j_{y} n_{x}$; it is the so-called pseudoscalar. Thus, the $j_{\varphi}$-component of the induced current is determined as follows:

$j_{\varphi}(\mathbf{r})=-\frac{e}{2} \int_{C} \frac{\mathrm{d} E}{2 \pi i} \operatorname{tr} G\left(\mathbf{r}, \mathbf{r}^{\prime} ; E\right) \gamma_{\varphi}$.

For the Dirac equation in a Coulomb field in $3+1$ dimensions, the radial partial Green function is given by [39]

$$
\begin{aligned}
G_{l}\left(r, r^{\prime} ; E\right) \gamma^{0}= & \frac{1}{\mathrm{~W}(E)}\left[\Theta\left(r^{\prime}-r\right) \psi_{R}(r) \psi_{I}^{\dagger}\left(r^{\prime}\right)\right. \\
& \left.+\Theta\left(r-r^{\prime}\right) \psi_{I}(r) \psi_{R}^{\dagger}\left(r^{\prime}\right)\right],
\end{aligned}
$$

where $\mathrm{W}(E)$ is the Wronskian and $\psi_{R}(r)$ and $\psi_{I}(r)$ are the regular and irregular solutions of the radial Dirac equation $(\check{H}-E) \psi(r)=0$. One can show that the Green function in our case can be represented via the regular and irregular solutions of the two-dimensional radial Dirac equation, just as in $3+1$ dimensions. It is convenient to apply the doublet (11) as the regular solutions and (14) as the irregular ones. In such a way, we first construct the Green function for the model with the nonzero (at $r<R$ ) AB potential by analogy with the Coulomb case in $3+1$ dimensions. The $(r-$ independent) Wronskian, defined by two doublets (11) and (14) as $\operatorname{Wr}(V, F)=r V i \sigma_{2} F=r\left(v_{1} f_{2}-f_{1} v_{2}\right)$, is easily calculated to be

$\mathrm{Wr}(V, F)=\mp A C \lambda^{-v} p^{v}$.

Here the upper (lower) signs should be taken for $l+\mu>0$ $(l+\mu<0)$.

For the induced vacuum charge and current in the $A B$ potential, we obtain

$$
\begin{aligned}
j_{0}(r)= & -e N^{2}\left[K_{\beta}^{2}(x)+K_{1-\beta}^{2}(x)\right]_{\beta>1 / 2} \\
& -e \int_{C_{-}+C_{+}} \frac{\mathrm{d} E}{4 \pi^{2} i} \operatorname{tr}\left[\lambda ^ { v } p ^ { - v } \left((E+m) K_{v}(\lambda r) J_{v}(p r)\right.\right. \\
& -s \sqrt{(m-E)(E-m)}\left(-K_{v}^{\prime}(\lambda r) \pm s \frac{v}{\lambda r} K_{v}(\lambda r)\right) \\
& \left.\left.\times\left(\mp s J_{v}^{\prime}(p r)+\frac{v}{p r} J_{v}(p r)\right)\right)\right]
\end{aligned}
$$


and

$$
\begin{aligned}
j_{\varphi}(r)= & -2 e N^{2}\left[K_{\beta}(x) K_{1-\beta}(x)\right]_{\beta>1 / 2} \\
& -e \int_{C_{-}+C_{+}} \frac{\mathrm{d} E}{4 \pi^{2} i} t r \\
& \times\left[\lambda ^ { v } p ^ { - v } \left(p K_{v}\left(\mp s J_{v}^{\prime}(p r)+\frac{v}{p r} J_{v}(p r)\right)+\right.\right. \\
& \left.\left.+s \lambda J_{v}(p r)\left(-K_{v}^{\prime}(\lambda r) \pm s \frac{v}{\lambda r} K_{v}(\lambda r)\right)\right)\right] .
\end{aligned}
$$

In (30) and (31) $N$ is the normalization factor, the prime denotes the derivative of function with respect to argument and $t r \equiv \sum_{l=-\infty}^{\infty} \sum_{s= \pm 1} ; x=\lambda(\beta, R) r$, where $\lambda(\beta, R)$ is determined by (22) so $x \sim c(r / R), c \sim 1$.

Using the recurrent relations for the Bessel functions and summing over $s$, we find

$$
\begin{aligned}
j_{0}(r)= & -e N^{2}\left[K_{\beta}^{2}(x)+K_{1-\beta}^{2}(x)\right]_{\beta>1 / 2} \\
& \pm e \int_{C_{-}+C_{+}} \frac{\mathrm{d} E}{4 \pi^{2} i} \sum_{l=-\infty}^{\infty} \lambda^{v} p^{-v}(E+m) K_{\nu}(\lambda r) J_{v}(p r)
\end{aligned}
$$

and

$$
\begin{aligned}
j_{\varphi}(r)= & -2 e N^{2}\left[K_{\beta}(x) K_{1-\beta}(x)\right]_{\beta>1 / 2} \\
& -e \int_{C_{-}+C_{+}} \frac{\mathrm{d} E}{4 \pi^{2} i} \sum_{l=-\infty}^{\infty} \lambda^{v} p^{-v} \frac{v}{r} K_{v}(\lambda r) J_{v}(p r) .
\end{aligned}
$$

The integral over $E$ from the first term of integrand (32) gives 0 , because integrals taken along the paths $C_{-}$and $C_{+}$cancel in pairs. Now, it is possible to deform the paths $C_{-}$and $C_{+}$ to the imaginary $E$ axis:

$$
\begin{aligned}
j_{0}(r)= & -e N^{2}\left[K_{\beta}^{2}(x)+K_{1-\beta}^{2}(x)\right]_{\beta>1 / 2} \\
& \pm e m \int_{-\infty}^{\infty} \frac{\mathrm{d} E}{4 \pi^{2}} \sum_{l=-\infty}^{\infty} K_{v}(z) J_{v}(z)=j_{0}^{b}(r)+j_{0}^{v}(r)
\end{aligned}
$$

and

$$
\begin{aligned}
j_{\varphi}(r)= & -2 e N^{2}\left[K_{\beta}(x) K_{1-\beta}(x)\right]_{\beta>1 / 2} \\
& -\frac{e}{r} \int_{-\infty}^{\infty} \frac{\mathrm{d} E}{4 \pi^{2}} \sum_{l=-\infty}^{\infty} \nu K_{v}(z) I_{v}(z)=j_{\varphi}^{b}(r)+j_{\varphi}^{v}(r) .
\end{aligned}
$$

Here, the symbols $b$ and $v$ characterize the contributions from the bound and the free states, respectively. In (34) the upper (lower) signs should be taken for $l+\mu>0(l+\mu<0)$, $I_{v}(z)=e^{-i \pi v / 2} J_{v}(i z)$ is the modified Bessel function of the first kind of the argument $z=\sqrt{m^{2}+E^{2}} r$. An essential detail is that the vacuum charge $\left(j_{0}^{b}(r)\right)$ and the current $\left(j_{\varphi}^{b}(r)\right)$ are singular at the origin, localized near the point $r=0$, and exponentially small at $r \gg R$ :

$j_{0}^{b}(r), \quad j_{\varphi}^{b}(r) \sim \frac{e}{r} e^{-c r / R}$.

Summation over $l$ in (34) gives $j_{0}^{v}(r)=0$. For massless fermions, this result was obtained and explained in [20] as follows: as far as $j_{0}^{v}(r)$ should be an odd function of $\mu$ due to the Furry theorem, it must be a pseudoscalar, which would contradict to parity conservation of the two-dimensional Dirac equation for a massless particle. In the two-dimensional model with mass term, this term is not invariant with respect to the operations of time inversion and of spatial parity. Nevertheless, the nonzero vacuum charge density $j_{0}^{b}(r)$ appears due to the vacuum charge of the bound (empty!) state; the vacuum charge spatial distribution is defined by the modulus squared of the fermion wave function in the bound state (with $\beta>1 / 2$ ). The vacuum current density contains the vacuum currents $j_{\varphi}^{b}(r)$ and $j_{\varphi}^{v}(r)$ that are, respectively, due to the vacuum current of bound state and the vacuum current of free (filled!) states $j_{\varphi}^{v}(r)$, which is spread out over an energy range of the negative energy continuum. It is worth to note that the vacuum charge density $j_{0}^{v}(\mathbf{r})$ is induced by the homogeneous background magnetic field in the massive $\mathrm{QED}_{2+1}[6,7]$, but this is not so in the $\mathrm{QED}_{3+1}$.

Using the easily checked representation

$K_{v}(z) I_{v}(z)=\int_{0}^{\infty} \mathrm{d} x e^{-2 z \cosh x} I_{2 v}(2 z \sinh x)$

and replacing variable $x$ by $y$ according to $\sinh x=\sinh ^{-1} y$, we obtain

$$
\begin{aligned}
j_{\varphi}^{v}(r)= & -\frac{e}{2 \pi^{2} r} \int_{0}^{\infty} \mathrm{d} E \sum_{l=-\infty}^{\infty} v \\
& \times \int_{0}^{\infty} \frac{\mathrm{d} y}{\sinh y} e^{-2 z \operatorname{coth} y} I_{2 v}(2 z / \sinh y) .
\end{aligned}
$$

At $m=0$ (37) takes the form

$$
\begin{aligned}
j_{\varphi}^{v}(r)= & -\frac{e}{2 \pi^{2} r} \int_{0}^{\infty} \mathrm{d} E \sum_{l=-\infty}^{\infty} v \\
& \times \int_{0}^{\infty} \frac{\mathrm{d} y}{\sinh y} e^{-2 E r \operatorname{coth} y} I_{2 v}(2 E r / \sinh y),
\end{aligned}
$$

which coincides exactly with that for the induced vacuum current of massless fermions for the first time obtained in [20].

The integrals over $y$ from any summand of (38) diverge, so some quantity $\delta \ll 1$ should be introduced as a lower limit of integration over $y$ in order for us to be able to change the 
order of summation and integration [20]. After that, we first take the integral over $E$ by means of the formula [39]

$$
\int_{0}^{\infty} \mathrm{d} t e^{-a t} I_{\nu}(b t)=\frac{b^{\nu}}{\sqrt{a^{2}-b^{2}}\left(a+\sqrt{a^{2}-b^{2}}\right)^{v}}
$$

As a result, we obtain

$$
j_{\varphi}^{v}(r)=-\frac{e}{2(\pi r)^{2}} \sum_{l=-\infty}^{\infty}|l+n+\beta| \int_{0}^{\infty} \frac{\mathrm{d} y}{\sinh y} e^{-2|l+n+\beta| y} .
$$

Taking the sum over $l$ by means of

$$
\begin{aligned}
& 4 \sum_{k=1}^{\infty} k e^{-2 k y}=-\cosh ^{-2} y \\
& 2 \sum_{k=1}^{\infty} e^{-2 k y}=\left(e^{y} / \cosh y\right)-2
\end{aligned}
$$

and then the integral over $y$ using the formula [39]

$$
\int_{0}^{\infty} \mathrm{d} t \frac{\sinh a t}{\sinh b t}=\frac{\pi}{2 b} \tanh \frac{a \pi}{2 b}, \quad b>|a|,
$$

we finally arrive at
If we take a real solenoid, then to learn the role of a finite small radius $R$ we consider again the semirealistic model with the nonzero AB potential at $r<R$. For such a model we can use a linear combination of the $J_{v}(p r)$ and $J_{-v}(p r)$ Bessel functions but not the Bessel $\left(J_{|l| \pm s}(p r)\right)$ and Neumann $\left(N_{|l| \pm s}(p r)\right)$ functions as the $\psi_{R}, \psi_{I}$ solutions for $r<R$. For $r>R$ the $K_{v}, I_{v}$ modified Bessel functions apply. Then continuity at $r=R$ determines the linear combination of the $J_{v}$ and $J_{-v}$ solutions that joins the $K_{v}$ function giving the irregular solution. Continuity at $r=R$ also can determine the particular linear combination of the $K_{v}$ and $I_{v}$ that joins the regular solution (i.e., one integrable near $r=0$ !). With such constructed solutions the Green function will receive finite-size corrections as functions at $r<R$ and $r>R$ [39]. It is wonderful that for the considered model we do not need to construct the regular solutions in the range $r<$ $R$ because we can apply regular solutions constructed by means of the self-adjoint method in [42]. Regular solutions (doublets) must satisfy the so-called self-adjoint boundary conditions [16],

$$
\left.\left(F^{\dagger}(r) i \sigma_{2} F(r)\right)\right|_{r=0}=\left.\left(\bar{f}_{1} f_{2}-\bar{f}_{2} f_{1}\right)\right|_{r=0}=0 .
$$

Physically, the self-adjoint boundary conditions show that the radial component of probability current density is equal to zero at the origin (the "origin" does not produce particles). For our problem the needed regular solution has the form

$\psi(r)=\left(\begin{array}{l}f_{1}(p r) \\ f_{2}(p r)\end{array}\right)=D\left(\begin{array}{c}\sqrt{E+m}\left[\cos (\theta / 2) J_{(1+s) / 2-s \beta}(p r)-\sin (\theta / 2) J_{-(1+s) / 2+s \beta}(p r)\right] \\ -s \sqrt{E-m}\left[\cos (\theta / 2) J_{(s-1) / 2-s \beta}(p r)+\sin (\theta / 2) J_{(1-s) / 2+s \beta}(p r)\right]\end{array}\right)$,

$j_{\varphi}^{v}(r)=\frac{e}{4 \pi r^{2}}(2|\beta|-1)^{2} \tanh \pi|\beta|$.

We emphasize that $j_{\varphi}^{b}(r)=0$ at $m=0$, because the normalization constant $N \sim \sqrt{m}$. A massless charged fermion cannot be bound with an $\mathrm{AB}$ potential. As far as the main contribution to the integral over $E$ at $r \gg 1 / m$ is given by the region $E \sim 1 / r$, one can expect that Eq. (43) can be used for an estimation of the massive case if we replace $r^{2}$ by $r^{2} \sqrt{1+(m r)^{2}}$ in the denominator of (43).

One can see that the induced current depends only on the fractional part $\beta$ of $\mu$ and is a finite periodical function of the magnetic flux. For the first time these results were obtained in [20].

It should be noted that the nonzero probability current arises under the effect of a constant uniform magnetic field on an electron bound by an attractive delta-function potential $[40,41]$. The spatial distribution of the probability current density resembles the spatial induced vacuum current $j_{\varphi}^{b}(r)$, but the $j_{\varphi}^{b}(r)$ current arises due to vacuum polarization, i.e. when the bound state is not filled. We also note that the vacuum polarization must manifest itself in such a way so as to modify (change) the external potential. where $D$ is the normalization factor and, as above, $1>\beta>$ $0, \xi=\tan (\theta / 2)$. Note that the correct values of the selfadjoint parameter determine the behavior of the upper (lower) component of the doublet (45) at the origin. Particularly, the case $\theta=0(\theta=\pi)$ is equivalent to insisting that the upper (lower) component stays regular at the origin for any $s= \pm 1$ and, generally speaking, for $\mu>0$. If $\theta \neq 0, \pi$ both components of the doublet contain singular terms at the origin.

We need to estimate the contribution of the correction functions to the induced vacuum current. To estimate the Green correction function at $r \gg R$ we do not need to sum over $l$ and $s$; it hence suffices to consider only the case, for example, $s=-1$. First, from the simple system

$$
\begin{aligned}
& C_{1} K_{\beta}(z)+C_{2} I_{\beta}(z) \\
& \left.\quad=(\theta / 2) J_{\beta}(p R)-\sin (\theta / 2) J_{-\beta}(p R)\right] \equiv f_{1} \\
& C_{1} K_{1-\beta}(z)+C_{2} I_{1-\beta}(z)=(\theta / 2) J_{\beta-1}(p R)+\sin (\theta / 2) \\
& \left.J_{1-\beta}(p R)\right] \equiv f_{2}, \quad z=\sqrt{m^{2}+E^{2}} R
\end{aligned}
$$

we obtain

$C_{1}=\frac{f_{1} I_{1-\beta}-f_{2} I_{\beta}}{W}, \quad W=K_{\beta} I_{1-\beta}-K_{1-\beta} I_{\beta}$. 
At $r \gg R$ the contribution of the Green (singular) correction function to the integrand in Eq. (33) is determined with $C_{1}(E R)$. Estimating $C_{1}$ with using Eq. (18), one can obtain $C_{1}(E R) \sim(E R)^{2} \cos (\theta / 2),(E R)^{2 \beta} \sin (\theta / 2)$. The main contribution to the integral over $E$ at $r \gg R$ is given by $E \sim 1 / r$, so that the contribution of the Green correction function to the induced current is suppressed by the factors $(R / r)^{2} \cos (\theta / 2),(R / r)^{2 \beta} \sin (\theta / 2)$. These results are in agreement with estimations made in [20]. The contribution of the Green (singular) correction function to the vacuum charge density $j_{0}^{v}(r)$ may be nonzero and may contain the same factors.

\section{Summary}

We have investigated the effect of vacuum polarization for charged massive fermions in an $\mathrm{AB}$ potential in $2+1$ dimensions using the causal Green function of the Dirac equation with the $\mathrm{AB}$ potential represented via the regular and irregular solutions of the two-dimensional radial Dirac equation, which takes into account the fermion spin. It is shown that the vacuum current density contains the contribution from free filled states of the negative energy continuum as well as that from a bound (empty!) state, which can emerge in the above background due to the interaction of the fermion's spin magnetic moment with the $\mathrm{AB}$ magnetic field, while the nonzero vacuum charge density appears only due to the contribution from the bound state. We have derived (recovered for massless fermions) expressions for the vacuum charge and vacuum current densities for graphene in the field of an infinitesimally thin solenoid perpendicular to the plane of a sample.

Periodicity of the $j_{\varphi}^{v}$ vacuum electric current due to vacuum polarization was observed recently in [43] in "a quantum-tunneling system using two-dimensional ionic structures in a linear Paul trap". "The charged quantumtunneling particles should be affected by the vector potential of a magnetic field throughout the entire process, even during quantum tunneling, and, thus, the AB effect should occur for tunneling particles". The authors "were successful in observing the AB effect of tunneling particles using this system". It was revealed that "the tunneling rate of the structure periodically depends on the strength of the magnetic field, whose period is the same as the magnetic-flux quantum $\phi_{0}$ ". It will be noted that this result is in agreement with Eq. (43), which is a periodical function of the magnetic flux.

The work was supported in part by the Ministry of Education and Science of the Russian Federation grant (Agreement No 14.576.21.0025 of 27.07.2014).
Open Access This article is distributed under the terms of the Creative Commons Attribution License which permits any use, distribution, and reproduction in any medium, provided the original author(s) and the source are credited

Funded by $\mathrm{SCOAP}^{3}$ / License Version CC BY 4.0.

\section{References}

1. Y. Aharonov, D. Bohm, Phys. Rev. 115, 485 (1959)

2. R.E. Prange, S.M. Girvin (eds.), The Quantum Hall Effect, 4th edn (Springer, New York, 1990)

3. F. Wilczek, Fractional Statistics and Anyon Superconductivity (World Scientific, Singapore, 1990)

4. C.R. Hagen, Phys. Rev. Lett. 64, 503 (1990)

5. M.G. Alford, F. Wilczek, Phys. Rev. Lett. 62, 1071 (1989)

6. A.J. Niemi, G.W. Semenoff, Phys. Rev. Lett. 51, 2077 (1983)

7. V.R. Khalilov, Theor. Math. Phys. 125, 1413 (2000)

8. K.S. Novoselov et al., Science 306, 666 (2004)

9. A.H. Castro, Neto et al., Rev. Mod. Phys. 81, 109 (2009)

10. N.M.R. Peres, Rev. Mod. Phys. 82, 2673 (2010)

11. V.N. Kotov et al., Rev. Mod. Phys. 84, 1067 (2012)

12. K.S. Novoselov et al., Nature 438, 197 (2005)

13. Z. Jiang, Y. Zhang, H.L. Stormer, P. Kim, Phys. Rev. Lett. 99, $106802(2007)$

14. I.S. Terekhov, A.I. Milstein, V.N. Kotov, O.P. Sushkov, Phys. Rev. Lett. 100, 076803 (2008)

15. F. Guinea, M.I. Katsnelson, A.K. Geim, Nat. Phys. 6, 30 (2009)

16. V.M. Pereira, J. Nilsson, A.H. Castro Neto, Phys. Rev. Lett. 99, $166802(2007)$

17. A.V. Shytov, M.I. Katsnelson, L.S. Levitov, Phys. Rev. Lett. 99, 236801 (2007)

18. K. Nomura, A.H. MacDonald, Phys. Rev. Lett. 98, 076602 (2007)

19. I.F. Herbut, Phys. Rev. Lett. 104, 066404 (2010)

20. R. Jackiw, A.I. Milstein, S.-Y. Pi, I.S. Terekhov, Phys. Rev. B 80, 033413 (2009)

21. Yu.A. Sitenko, Phys. Rev. D 60, 125017 (1999)

22. Yu.A. Sitenko, Ann. Phys. 282, 167 (2000)

23. B.L. Voronov, D.M. Gitman, I.V. Tyutin, Theor. Math. Phys. 150, 34 (2007)

24. D.M. Gitman, I.V. Tyutin, B.L. Voronov, Self-Adjoint Extensions in Quantum Mechanics (Springer Science+Business Media, New York, 2012)

25. V.R. Khalilov, K.-E. Lee, J. Phys. A 44, 205303 (2011)

26. V.R. Khalilov, Theor. Math. Phys. 175, 637 (2013)

27. V.R. Khalilov, Eur. Phys. J. C 73, 2548 (2013)

28. V.R. Khalilov, Eur. Phys. J. C 74, 2708 (2014)

29. Ph. Gerbert, Phys. Rev. D 40, 1346 (1989)

30. M.G. Alford, J. March-Pussel, F. Wilczek, Nucl. Phys. B 328, 140 (1989)

31. V.R. Khalilov, C.-L. Ho, Ann. Phys. 323, 1280 (2008)

32. E.O. Silva, F.M. Andrade, C. Filgueiras, H. Belich, Eur. Phys. J. C 73(4), 2402 (2013)

33. Y. Hosotani, Phys. Lett. B 319, 332 (1993)

34. F.M. Andrade, E.O. Silva, M. Pereira, Phys. Rev. D 85(4), 041701(R) (2012)

35. F.M. Andrade, E.O. Silva, Phys. Lett. B 719(4-5), 467 (2013)

36. E.O. Silva, F.M. Andrade, Europhys. Lett. 101(5), 51005 (2013)

37. C. Filgueiras, E.O. Silva, F.M. Andrade, J. Math. Phys. 53(12), $122106(2012)$

38. V.R. Khalilov, K.-E. Lee, Mod. Phys. Lett. A 26(12), 865 (2011)

39. W. Greiner, J. Reinhardt, Quantum Electrodynamics, 4th edn. (Springer, Berlin, 2009) 
40. F.Kh. Chibirova, V.R. Khalilov, Mod. Phys. Lett. A 20, 663 (2005)

41. V.R. Khalilov, F.Kh. Chibirova, J. Phys. A Math. Theor. 40, 6469 (2007)
42. V.R. Khalilov, K.-E. Lee, I.V. Mamsurov, Mod. Phys. Lett. A 27(5), 1250027 (2012)

43. A. Noguchi, Y. Shikano, K. Toyoda, S. Urabe, Nat. Commun. 5, 3868 (2014) 